\title{
Análisis de las variantes anatómicas rinosinusales encontradas en pacientes con rinosinusitis crónica sometidos a cirugía funcional de nariz y senos paranasales
}

\author{
Analysis of the anatomical rhinosinusal variants found in patients with chronic \\ rhinosinusitis undergoing functional surgery of nose and paranasal sinuses
}

Anelia Baqueiro-Achach, ${ }^{*}$ Salomón Waizel-Haiał ${ }^{\ddagger}$

\begin{abstract}
RESUMEN
Introducción: La rinosinusitis crónica es una enfermedad de alta prevalencia mundial cuyo tratamiento inicial es el manejo médico a base de esteroides e irrigaciones nasales; varios pacientes no logran control adecuado, por lo que son candidatos a cirugía funcional endoscópica. Se ha identificado una gran cantidad de variantes anatómicas rinosinusales, las cuales podrían predisponer al fracaso del manejo médico. Objetivo: Identificar las variantes anatómicas en pacientes con rinosinusitis crónica sometidos a cirugía funcional endoscópica de nariz y senos paranasales en un hospital de tercer nivel de atención. Material y métodos: Se realizó un estudio retrospectivo, transversal, descriptivo y observacional. Se revisaron expedientes clínicos de pacientes con diagnóstico de rinosinusitis crónica sometidos a cirugía funcional endoscópica en el periodo de enero a diciembre de 2017; se analizaron los estudios de tomografía computarizada prequirúrgicos para identificar las variantes anatómicas presentes, así como la severidad de la rinosinusitis utilizando el sistema de clasificación tomográfica de Lund-Mackay. Resultados: Se encontraron variantes anatómicas en $85.1 \%$ de los pacientes, la más frecuente fue la desviación septal (76.5\%). Conclusión: No hay relación importante entre la severidad de la enfermedad y la configuración anatómica del paciente.
\end{abstract}

Palabras clave: Rinosinusitis crónica, cirugía funcional de nariz y senos paranasales.

Nivel de evidencia: III

\begin{abstract}
Introduction: Chronic rhinosinusitis is a high global prevalence disease which initial treatment is medical management based on steroids and nasal irrigations; many patients do not achieve adequate control, so they are candidates to functional endoscopic surgery. Many rhinosinusal anatomical variants have been identified, which could predispose failure of medical management. Objective: To identify the anatomical variants in patients with chronic rhinosinusitis undergoing functional endoscopic surgery of the nose and paranasal sinuses in a tertiary care hospital. Material and methods: A retrospective, cross-sectional, descriptive and observational study was carried out. Clinical records of patients diagnosed with chronic rhinosinusitis undergoing functional endoscopic surgery in the period from January to December 2017 were studied; pre-surgical computed tomography studies were reviewed to identify the anatomical variants present as well as the severity of rhinosinusitis using the Lund-Mackay tomographic classification system. Results: Anatomical variants were found in $85.1 \%$ of the patients; the most frequent was septal deviation (76.5\%). Conclusion: There is no important relationship between the severity of the disease and the anatomical configuration of the patient.
\end{abstract}

Keywords: Chronic rhinosinusitis, functional endoscopic sinus surgery.

Level of evidence: III

\footnotetext{
* Servicio de Otorrinolaringología.

‡ Jefe de Servicio de Otorrinolaringología.
}

Unidad Médica de Alta Especialidad, Hospital de Especialidades Centro Médico Nacional Siglo XXI. Ciudad de México, México.
Correspondencia: Dr. Salomón Waizel-Haiat

Prol. Vasco de Quiroga Núm. 4001-802, Juan Salvador Agraz,

Santa Fe, Ciudad de México.

E-mail: swaizel@hotmail.com

Este artículo puede ser consultado en versión completa en: www.medigraphic.com/analesmedicos 


\section{INTRODUCCIÓN}

La rinosinusitis se define como la inflamación sintomática de la mucosa de la nariz y los senos paranasales. Se estima que uno de cada ocho adultos en Estados Unidos de América es afectado, lo que se traduce en 30 millones de pacientes diagnosticados anualmente y un costo directo del manejo que excede los 11 billones de dólares al año, además de los gastos generados por la disminución en la calidad de vida y productividad en el trabajo medida por el ausentismo y reducción en el rendimiento laboral. ${ }^{1,2}$

Según su duración, se puede clasificar en rinosinusitis aguda (RSA) si es menor de cuatro semanas o rinosinusitis crónica (RSC) si es mayor de 12 semanas, con o sin exacerbaciones agudas. Algunas veces la rinosinusitis de duración entre cuatro y 12 semanas se llama subaguda.

Diferentes autores han evaluado la relación entre variantes anatómicas rinosinusales y la incidencia de rinosinusitis. Aunque la fisiopatología y etiología exactas de la enfermedad no se conocen en su totalidad, la obstrucción del complejo osteomeatal es parte primordial de los mecanismos causales de la inflamación; en teoría cualquier alteración en la anatomía que condicione mayor obstrucción en el mismo propicia esta inflamación. Existen determinadas variantes anatómicas, principalmente de la pared lateral nasal, que pueden contribuir a la obstrucción del drenaje y ventilación del complejo osteomeatal. ${ }^{3}$ Algunas asociadas con rinosinusitis crónica son: desviación septal, concha bullosa, cornete medio paradójico, bulla etmoidal hipertrófica, medialización o lateralización del proceso uncinado, celdilla de Haller, celdilla de Onodi. ${ }^{3,4}$

La incidencia de estas variantes tanto en la población sana como en los pacientes con rinosinusitis crónica no está bien establecida, por lo tanto su rol en la patogénesis de la enfermedad permanece incierto.

La inflamación de la mucosa debe documentarse y confirmarse con algún hallazgo para poder integrar el diagnóstico de rinosinusitis. Una manera de hacerlo es a través de la tomografía computarizada en la que se puede cuantificar la extensión de la enfermedad inflamatoria, la cual se basa en la opacidad de los senos paranasales, además de mostrar anomalías que condicionen obstrucción del complejo osteomeatal, variantes anatómicas y poliposis nasosinusal; también debe realizarse cuando se planea algún procedimiento quirúrgico endoscópico. ${ }^{1,5}$

Kennedy ${ }^{6}$ propuso la necesidad de un sistema de clasificación para determinar la extensión y se- veridad de la enfermedad nasosinusal; los hallazgos tomográficos no correlacionan necesariamente con los síntomas ni gravedad, pero ofrecen un método objetivo para controlar la enfermedad recurrente o crónica. ${ }^{1}$

Existen varios sistemas para correlacionar los hallazgos tomográficos con la severidad de la enfermedad; el de Lund-Mackay es uno de los más utilizados en ensayos clínicos además de ser el que tiene la mejor concordancia interobservador e intraobservador. ${ }^{7,8}$ Este método fue creado a mediados de los años 80 , y uno de sus objetivos es ser una herramienta para facilitar la toma de decisiones terapéuticas. Se realiza mediante la evaluación tomográfica de los senos paranasales. A cada grupo se le asigna una calificación según la opacidad que muestre cada seno paranasal: 0 ausencia completa, 1 opacidad parcial y 2 opacidad total; se evalúa el seno maxilar, celdillas etmoidales anteriores, celdillas etmoidales posteriores, seno esfenoidal, seno frontal y complejo osteomeatal, al final los valores de cada lado se suman y se obtiene un resultado entre 0 y $24 .{ }^{7,9}$

Con relación al manejo, el tratamiento médico sigue siendo la base, principalmente con esteroides tópicos e irrigaciones nasales, éste es indispensable incluso en pacientes sometidos a tratamiento quirúrgico tanto en el preoperatorio como postoperatorio.

La cirugía funcional de nariz y senos paranasales (FESS, por sus siglas en inglés) se propone como manejo para la RSC refractaria a tratamiento médico; es una de las intervenciones quirúrgicas que con más frecuencia se practica en Norteamérica; se estima que 450,000 casos de FESS se realizan anualmente sólo en los Estados Unidos de América. ${ }^{10}$

Se ha comparado el resultado de la cirugía endoscópica con el tratamiento médico en pacientes con RSC refractaria, analizado el costo/efectividad de cada una y se ha encontrado que la calidad de vida es mejor en los pacientes sometidos a cirugía endoscópica. ${ }^{11,12}$ A pesar de que el costo del procedimiento quirúrgico es mayor, con el tiempo los análisis probabilísticos han revelado que la cirugía endoscópica se convierte en una intervención rentable en el tercer año posterior a la misma. ${ }^{2}$ La cirugía per se no resuelve la inflamación asociada con la rinosinusitis crónica, la atención postoperatoria y el manejo médico continuo son fundamentales para el éxito de estos pacientes, a pesar de esto, un pequeño subconjunto de pacientes presentará síntomas refractarios tanto en las terapias médicas como a las quirúrgicas. ${ }^{6,10}$

Objetivo: identificar las variantes anatómicas en pacientes con rinosinusitis crónica sometidos a ciru- 
gía funcional endoscópica de nariz y senos paranasales en un hospital de tercer nivel de atención.

\section{MATERIAL Y MÉTODOS}

El estudio realizado es de tipo retrospectivo, transversal, descriptivo y observacional, el universo fueron los pacientes con rinosinusitis crónica del servicio de otorrinolaringología de un hospital universitario de tercer nivel de atención en la Ciudad de México, sometidos a cirugía funcional de nariz y senos paranasales vía endoscópica en el periodo del 01 de enero al 31 de diciembre de 2017. Los criterios de inclusión fueron: 1 . Ser pacientes del servicio de otorrinolaringología del hospital con diagnóstico de rinosinusitis crónica. 2. Haber sido sometido a cirugía funcional de nariz y senos paranasales vía endoscópica en el periodo estudiado. Se excluyeron los pacientes con antecedente de cirugía nasal previa, así como aquéllos con diagnóstico de poliposis nasal masiva que imposibilitaba la adecuada visualización de las estructuras valoradas en la tomografía o diagnóstico de tumoración nasal. Fueron eliminados los que no contaron con tomografía computarizada en el sistema de archivo electrónico digital de imágenes del hospital o que carecían de expediente clínico completo. La muestra fue de 62 pacientes que cumplieron los criterios de inclusión. El muestreo fue no probabilístico.

Se analizaron los expedientes clínicos de los pacientes incluidos en el estudio, de igual modo se revisaron los estudios de tomografía computarizada prequirúrgicos para identificar la severidad de la rinosinusitis medida individualmente en cada seno paranasal mediante el sistema de Lund-Mackay ${ }^{9}$ y asimismo las variantes anatómicas presentes.

Con relación a los aspectos éticos, el estudio es considerado como investigación sin riesgo de acuerdo al artículo 17 del reglamento de la Ley General de Salud en Materia de Investigación para la Salud. La información obtenida se mantuvo en el anonimato y la confidencialidad, de acuerdo con los principios éticos para las investigaciones médicas en seres humanos del reglamento de la Ley General de Salud en Materia de Investigación para la Salud y la Declaración de Helsinki de la Asociación Médica Mundial. Para identificar los datos obtenidos se asignaron folios a cada paciente y así mantener el anonimato.

Para el procesamiento de la información se elaboró una base de datos en Excel ${ }^{\circledR}$ de Microsoft ${ }^{\circledR}, \mathrm{y}$ el análisis estadístico se realizó con el paquete computacional Statistical Package for the Social Sciences
(SPSS) ${ }^{\circledR}$ versión 24 . Se obtuvieron las estadísticas descriptivas de media, desviación estándar, valores mínimo y máximo a las variables edad y severidad de la rinosinusitis. A las variables de sexo, concha bullosa derecha e izquierda, cornete paradójico derecho e izquierdo, celdillas de Haller y de Onodi derecha e izquierda, grado de rinosinusitis en cada seno maxilar, celdillas etmoidales anteriores y posteriores, seno esfenoidal y frontal, así como complejo osteomeatal derecho e izquierdo se les obtuvo su tabla de distribución de frecuencia absoluta y relativa, medida esta última como proporción.

Con el objeto de detectar alguna asociación entre variables de variantes anatómicas y severidad de la rinosinusitis se obtuvo el coeficiente de Spearman.

\section{RESULTADOS}

Según los registros de procedimientos quirúrgicos realizados en el servicio de otorrinolaringología del hospital, en el año 2017 se efectuaron 62 cirugías funcionales endoscópicas de nariz y senos paranasales, de las cuales 47 fueron incluidas en este estudio, las 15 restantes fueron excluidas por no contar con tomografía computarizada para la evaluación o por presentar cirugía nasal previa o poliposis nasal masiva. Veintiséis pacientes fueron de sexo femenino $(55.3 \%)$ y 21 pertenecían al masculino (44.7\%). La edad media fue de 43 años, mínima 22 y máxima 70 años (desviación estándar de 13.15).

En cuanto a las variantes anatómicas, $85.1 \%$ de los pacientes $(n=40)$ mostró al menos una en la tomografía computarizada, siendo la desviación septal la más frecuente. Se identificó la presencia de una sola variante en 27 pacientes (57.4\%), y un máximo de cinco variantes en un paciente $(2.1 \%)$ (Tabla 1).

Se apreció desviación septal en 36 pacientes (76.5\%), la mayoría se encontró entre los 20 y 30 grados con una media de 25.55 y una desviación están-

Tabla 1: Número de variantes anatómicas por paciente encontradas en la tomografía computarizada.

\begin{tabular}{ccr}
\hline Número de variaciones & $\mathrm{n}$ (casos) & $\%$ \\
\hline 0 & 7 & 14.9 \\
1 & 27 & 57.4 \\
2 & 8 & 17.0 \\
3 & 3 & 6.4 \\
4 & 1 & 2.1 \\
5 & 1 & 2.1 \\
Total & 47 & 100.0 \\
\hline
\end{tabular}


An Med (Mex) 2019; 64 (4): 241-247

Tabla 2: Frecuencia según el tipo de variante anatómica.

\begin{tabular}{lcc}
\hline Tipo de variación anatómica & $\mathrm{n}$ (pacientes) & $\%$ \\
\hline Desviación septal & 36 & 76.5 \\
Concha bullosa & 7 & 14.9 \\
Cornete medio paradójico & 7 & 14.9 \\
Celdilla de Onodi & 7 & 14.9 \\
Celdilla de Haller & 4 & 8.5 \\
\hline
\end{tabular}

dar de 10.74. En los 11 pacientes restantes se detectó algún grado de desviación septal, no considerado relevante (menor de 10 grados), por lo que no se tomó en cuenta como variante anatómica.

La presencia de las demás variantes anatómicas se describe en la Tabla 2.

Con relación a la severidad de la rinosinusitis, se utilizó el sistema tomográfico de Lund-Mackay ${ }^{9}$ (0-24 puntos). Se apreciaron grados de severidad muy variados, con una media de 12.49 y desviación estándar de 8.1; se encontraron desde pacientes que obtuvieron sólo un punto hasta pacientes con la calificación máxima de 24 puntos con todos los senos paranasales ocupados en su totalidad. El seno paranasal más afectado fue el maxilar ( $87.2 \%$ de los pacientes presentaron afección del lado derecho y $80.9 \%$ del izquierdo), seguido de las celdillas etmoidales anteriores $(76.6 \%$ de los pacientes mostraron afección del lado derecho y $83 \%$ del izquierdo). La mayor severidad se observó en el complejo osteomeatal izquierdo seguido de las celdillas etmoidales anteriores (Tabla 3).

Con el objeto de detectar una posible asociación entre la severidad de la rinosinusitis y el número de variantes anatómicas encontradas en los pacientes, se obtuvo el coeficiente de correlación rho de Spearman, el cual presentó un valor de $-0.369(\mathrm{p}=0.011)$, asimismo se realizó la gráfica de dispersión entre estas variables.

\section{DISCUSIÓN}

En relación con la epidemiología de la población estudiada, $55.3 \%$ fueron mujeres y $44.7 \%$ hombres; este dato es notable, ya que la bibliografía consultada refiere mayor prevalencia de la enfermedad en el sexo masculino (entre 60.7-66.6\%) en comparación con el femenino (33.3-39.3\%). ${ }^{3,13}$ La edad media fue similar a la descrita en estos estudios (entre $40 \mathrm{y}$ 45.5 años). ${ }^{3,14}$

Existe controversia en relación con la importancia clínica de las variantes anatómicas nasosinusales y su relación con la presencia y severidad de la rinosinusitis, por este motivo el objetivo principal del estudio fue identificar variantes anatómicas en la tomografía preoperatoria de los pacientes, mismas que se encontraron en $85.1 \%$ de éstos. Este porcentaje se ubica en el rango promedio reportado en otros estudios similares; aunque algunos mencionan prevalencias menores de $70 \%$ y otros incluso mayores de $90 \%,{ }^{3,13,15}$ existe variabilidad entre los criterios diagnósticos utilizados en los diferentes estudios, algunos son más estrictos que otros, lo que puede explicar el diferente rango de prevalencia.

En cuanto al número de variantes anatómicas por paciente, en este estudio la mayoría (57\%) presentó una sola variante anatómica. En la mayoría de las investigaciones consultadas no se específica a detalle el número de variantes anatómicas por paciente, sólo en una referencia se menciona que la mayoría de sus casos (52\%) tuvo dos o más variaciones y $41 \%$ tuvo una sola. ${ }^{13}$

Desviación septal: se estima que la presencia de desviación septal en la población general es de 20$30 \%$ y que la desviación severa es un factor que contribuye a la sinusitis crónica. En este estudio fue la variante anatómica más frecuente con $76.5 \%$ de los casos (36 pacientes). La revisión de la literatura en relación a esta variante es amplia; en la mayoría de los reportes también es la más frecuente $e^{3,13,15,16}$ con porcentajes similares al obtenido entre $80-82 \%$;13,15,16 sin embargo, hay estudios con frecuencias notablemente menores entre 36-47\%.4,17 Algunos de los estudios clasifican la desviación por grupos según el grado de ésta utilizando diferentes parámetros. En

Tabla 3: Grado de severidad según el sistema tomográfico de Lund-Mackay por cada seno paranasal.

\begin{tabular}{lccc}
\hline & \multicolumn{3}{c}{ Grado de severidad (\%) } \\
\cline { 2 - 4 } Seno paranasal & 0 & 1 & 2 \\
\hline Seno maxilar derecho & 12.8 & 61.7 & 25.5 \\
Seno maxilar izquierdo & 19.1 & 53.2 & 27.7 \\
Celdillas etmoidales anteriores derechas & 23.4 & 34.0 & 42.6 \\
Celdillas etmoidales anteriores izquierdas & 17.0 & 38.3 & 44.7 \\
Celdillas etmoidales posteriores derechas & 29.8 & 31.9 & 38.3 \\
Celdillas etmoidales posteriores izquierdas & 27.7 & 31.9 & 40.4 \\
Seno esfenoidal derecho & 53.2 & 21.3 & 25.5 \\
Seno esfenoidal izquierdo & 57.4 & 21.3 & 21.3 \\
Seno frontal derecho & 54.3 & 10.9 & 34.8 \\
Seno frontal izquierdo & 51.1 & 10.6 & 38.3 \\
Complejo osteomeatal derecho & 31.9 & 34.0 & 34.0 \\
Complejo osteomeatal izquierdo & 36.2 & 14.9 & 48.9 \\
\hline
\end{tabular}




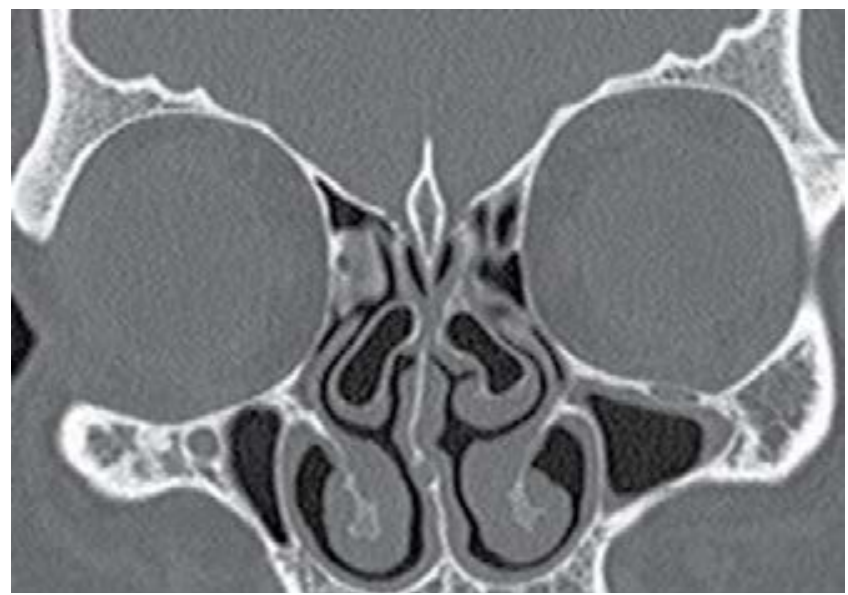

Figura 1: Presencia de concha bullosa bilateral.

nuestro caso, después de medir el grado de desviación se consideró como presencia de variante anatómica todas las desviaciones mayores de 10 grados sin clasificarlas en grupos.

Concha bullosa: en teoría la presencia de neumatización del cornete medio, conocida como concha bullosa, es una condición que puede bloquear el complejo osteomeatal y afectar el aclaramiento mucociliar del mismo causando rinosinusitis crónica, la presencia de algún grado de neumatización en la población general es de 11-15.9\%. Existen diferentes grados de neumatización y según el tamaño la afección puede variar. ${ }^{13,14,18}$ En esta investigación la frecuencia de concha bullosa (14.9\%) fue menor que la reportada en otros estudios, donde los porcentajes son muy diversos siendo todos mayores que el nuestro, con frecuencias que van desde $16.8 \%$ hasta $68 \% ; 4,19,20$ la mayoría con porcentajes cercanos a $30 \% .{ }^{13-17} \mathrm{La}$ variabilidad entre las frecuencias reportadas puede deberse a que varios autores consideran como concha bullosa cualquier grado de neumatización en el cornete medio, e incluso algunos no especifican el criterio utilizado. En este artículo se considera como concha bullosa a la neumatización de $50 \%$ o más de la porción vertical del cornete medio observada en la tomografía computarizada (Figura 1).

Cornete medio paradójico: la curvatura inversa del cornete medio, conocida como «cornete medio paradójico» (Figura 2), puede producir compresión del meato medio y obstrucción del drenaje. En nuestra investigación se encontraron siete casos de esta variante anatómica que representa $14.9 \%$, frecuencia que es similar a la descrita en la literatura (entre 10$14.4 \%)$, y que no varía de manera importante con la reportada en la población general (12.2\%).3,13,14,21
Celdillas de Haller: la presencia de celdillas etmoidales infraorbitarias, conocidas como «celdillas de Haller» fue descrita por dicho autor en el siglo XVIII; sin embargo, la definición actual es controversial. ${ }^{15}$ Algunos autores las consideran como celdillas etmoidales que se proyectan debajo de la bulla etmoidal dentro del piso orbitario, en la región de apertura del seno maxilar; otros las consideran como cualquier celdilla etmoidal que se encuentre en el piso de la órbita (definición utilizada en nuestro estudio), debido a lo cual, la frecuencia reportada puede variar de manera importante. Nosotros las identificamos en $8.5 \%$ de los pacientes, mientras que la literatura consultada refiere desde $3 \%{ }^{13,15}$ hasta $20 \%$ de rango promedio. ${ }^{3,22}$

Celdillas de Onodi: se refiere a aquella celdilla etmoidal posterior que se extiende lateral y/o superior al seno esfenoidal (Figura 3); su presencia aumenta la posibilidad de lesión a la arteria carótida interna y al nervio óptico durante la cirugía endoscópica nasosinusal. En nuestro estudio se logró identificar en $14.9 \%$ de los pacientes, lo cual coincide con las frecuencias reportadas en la literatura que van de 8.5-14\%. . $^{3,15,17,23}$

Con relación a la presencia y severidad de la rinosinusitis se observó que el seno paranasal más afectado fue el maxilar derecho en $87.2 \%$ de los pacientes; no obstante, la mayor severidad se encontró en el complejo osteomeatal izquierdo $(48.9 \%$ de los pacientes). Más allá de describir la presencia de esta patología, se intentó detectar una posible asociación entre el número de variantes anatómicas que mostraba cada paciente y la severidad de la rinosinusitis que padecía, para esto se obtuvo el coeficiente de correlación rho de Spearman, el cual tuvo un valor de

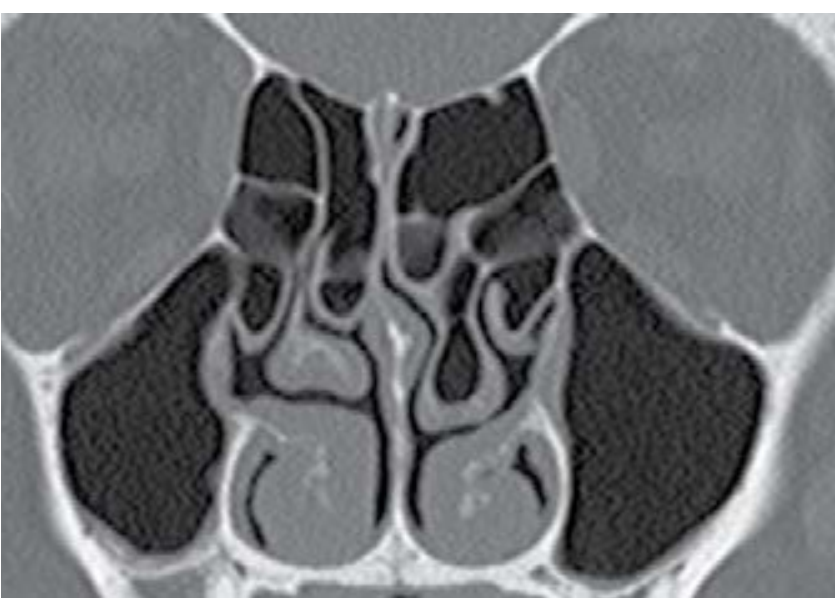

Figura 2: Cornete medio paradójico derecho y concha bullosa izquierda. 


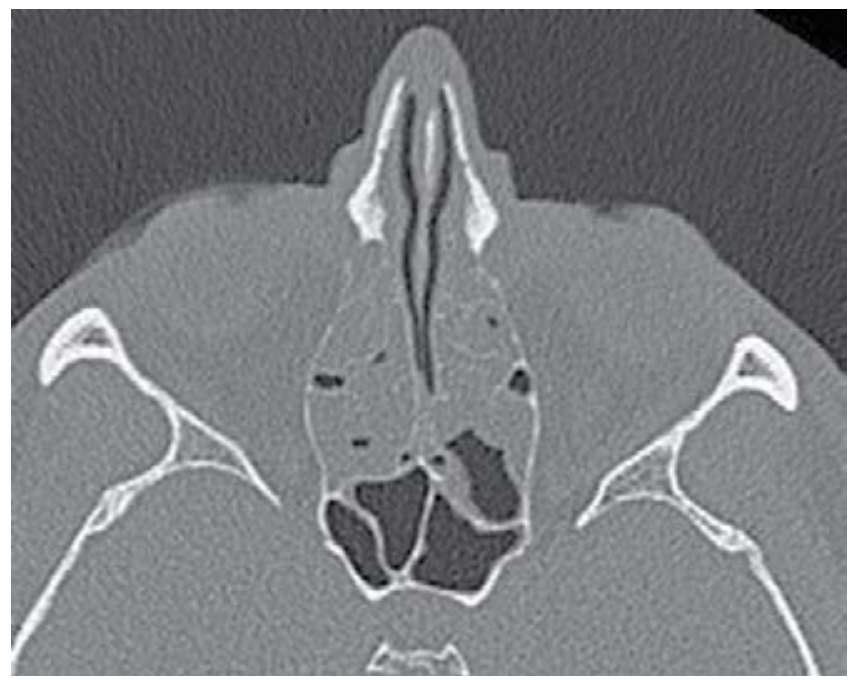

Figura 3: Celdilla de Onodi derecha.

$-0.369(\mathrm{p}=0.011)$, indicando que no podemos asociar el número de variantes anatómicas a un mayor grado de severidad en la rinosinusitis, incluso, según este valor existiría una asociación inversa media entre estas variables.

La literatura consultada no es concluyente para aceptar o rechazar esta asociación. Desde 1988, Stammberger y Wolf reportaron variantes en el cornete medio y proceso uncinado como posibles factores etiológicos de enfermedad sinonasal, ${ }^{24-27}$ y algunos autores han logrado correlacionarlo con valores estadísticamente significativos $;^{3}$ sin embargo, en la mayoría de los trabajos, incluido el nuestro, no se apreció diferencia significativa entre los pacientes que muestran variantes anatómicas nasosinusales y el grado de enfermedad que desarrollan. ${ }^{14,20} \mathrm{La}$ importancia de éstas va más relacionada al grado en el que contactan y obstruyen la mucosa del complejo osteomeatal. ${ }^{20,26}$

Identificar las variantes anatómicas presentes en cada paciente es indispensable en aquellos casos en los que se planea realizar cirugía endoscópica. ${ }^{20,26}$ En algunos casos pueden ser causas tratables de obstrucción del complejo osteomeatal, mientras que en otros conocerlas servirá para evitar o disminuir las complicaciones durante el procedimiento. ${ }^{28-30}$

\section{CONCLUSIONES}

Se encontraron variantes anatómicas rinosinusales en $85.1 \%$ de los pacientes con diagnóstico de rinosinusitis crónica sometidos a cirugía funcional endos- cópica de nariz y senos paranasales incluidos en el estudio. No se logró establecer una asociación estadísticamente significativa entre la severidad de la rinosinusitis y el número de variantes anatómicas presentes en cada paciente; la mayor importancia radica en el grado en el que estas variantes interfieren con el adecuado drenaje mucociliar, en particular del complejo osteomeatal.

En este sentido, la simple presencia de una variante no es suficiente para causar enfermedad si no interfiere con el drenaje de los senos paranasales.

Es indispensable una revisión detallada de las tomografías computarizadas preoperatorias para evitar complicaciones durante el procedimiento quirúrgico.

Se requiere la realización de un estudio en población sin presencia de rinosinusitis crónica para comparar la incidencia de variantes anatómicas entre sanos y enfermos.

\section{AGRADECIMIENTOS}

A las autoridades del Hospital de Especialidades del Centro Médico Nacional Siglo XXI del Instituto Mexicano del Seguro Social por proporcionar las facilidades para la realización de este estudio. Al doctor en ciencias José Waizel Bucay por los comentarios brindados y al maestro en ciencias José Luis Pablos Hach por su apoyo en el análisis estadístico.

\section{BIBLIOGRAFÍA}

1. Rosenfeld R, Piccirillo J, Chandrasekhar S, Brook I, Kumar AK, Kramper M et al. Clinical practice guideline (update): adult sinusitis. Otolaryngol Head Neck Surg. 2015; 152: S1-39.

2. DeCond A, Soler Z. Chronic rhinosinusitis: epidemiology and burden of disease. Am J Rhinol Allergy. 2016; 30 (2): 134-139.

3. Fadda GL, Rosso S, Aversa S, Petrelli A, Ondolo C, Succo G. Multiparametric statistical correlations between paranasal sinus anatomic variations and chronic rhinosinusitis. Acta Otorhinolaryngol Ital. 2012; 32 (4): 244-251.

4. Mohebbi A, Ahmadi A, Etemadi M, Safdarian M, Ghourchian S. An epidemiologic study of factors associated with nasal septum deviation by computed tomography scan: a cross sectional study. BMC Ear Nose Throat Disord. 2012; 12: 15.

5. Sharp H, Rowe-Jones J, Mackay I. The outcome of endoscopic sinus surgery: correlation with computerized tomography score and systemic disease. Clin Otolaryngol. 1999; 24 (1): 39-42.

6. Kennedy D. Novel options in the nonsurgical management of chronic rhinosinusitis. Int Forum Allergy Rhinol. 2018; 8 (4): 459-460.

7. Monteiro-Zappelini C, Rocha-Tavares H, Vega F, PicoliDanta I, Menino-Castilho A, Machado-de Carvalho G. Perfil tomográfico según la clasificación de Lund-Mackay de pacientes atendidos en una clínica ambulatoria de Otorrinolaringología. An Orl Mex. 2015; 60: 6-10.

8. Brooks S, Trope M, Blasetti M, Dogharamji LRN, Parasher A, glicksman TJ et al. Preoperative Lund-Mackay computed 
tomography score is associated with preoperative symptom severity and predicts quality-of-life outcome trajectories after sinus surgery. Int Forum Allergy Rhinol. 2018; 8 (6): 668-675.

9. Chen J, Chen D, Chen C. Lund-Mackay score for adult head and neck computed tomography. J Radiol Sci. 2011; 36: 203-208.

10. Schwartz J, Tajudeen B, Cohen N. Medical management of chronic rhinosinusitis - a review of traditional and novel medical therapies. Expert Opin Investig Drugs. 2017; 26 (10): 1123-1130.

11. Rudmik L, Smith LT, Schlosser R, Hwang P, Mace CJ, Soler MZ. Productivity costs in patients with refractory chronic rhinosinusitis. Laryngoscope. 2014; 124 (9): 2007-2012.

12. Rudmik L, Soler MZ, Mace J, Mace CJ, Schlosser JR, Smth LT. Economic evaluation of endoscopic sinus surgery versus continued medical therapy for refractory chronic rhinosinusitis. Laryngoscope. 2015; 125 (1): 25-32.

13. Kumarasekaran P, Yaadhavakrishnan RD, Sriraman G. Anatomical variations in patients with chronic sinusitis. Int $J$ Otorhinolaryngol Head Neck Surg. 2018; 4 (2): 428-431.

14. Cho J, Park MS, Chung Y, Seok-Chan H, Hyang Kwon K, Kook Kim J. Do anatomic variations of the middle turbinate have an effect on nasal septal deviation or paranasal sinusitis? Ann Otol Rhinol Laryngol. 2011; 120 (9): 569-574.

15. Pérez J, Carmona A, Herrera CJ, Jiménez J. Anatomical variations in the human paranasal sinus region studied by CT. J Anat. 2000; 197 (2): 221-227.

16. Kaplanoglu H, Kaplanoglu V, Dilli A, Toprak U, Hekimoğlu B. An analysis of the anatomic variations of the paranasal sinuses and ethmoid roof using computed tomography. Eurasian J Med. 2013; 45 (2): 115-125.

17. Arslan H, Aydinlioğlu A, Bozkurt M, Egeli E. Anatomic variations of the paranasal sinuses: CT examination for endoscopic sinus surgery. Auris Nasus Larynx. 1999; 26 (1): 39-48.

18. Roozbahany NA, Nasri S. Nasal and paranasal sinus anatomical variations in patients with rhinogenic contact point headache. Auris Nasus Larynx. 2013; 40 (2): 177-183.

19. Chaiyasate S, Baron I, Clement P. Analysis of paranasal sinus development and anatomical variations: a CT genetic study in twins. Clin Otolaryngol. 2007; 32 (2): 93-97.
20. San T, Erdoğan B, Taşel B. Triple-divided concha bullosa: a new anatomic variation. Case Rep Otolaryngol. 2013; 2013: 342615.

21. Sirikçi A, Bayazit YA, Bayram M, Kanlikama M. Ethmomaxillary sinus: a particular anatomic variation of the paranasal sinuses. Eur Radiol. 2004; 14 (2): 281-285.

22. Kantarci M, Karasen RM, Alper F, Onbas O, Okur A, Karaman A. Remarkable anatomic variations in paranasal sinus region and their clinical importance. Eur J Radiol. 2004; 50 (3): 296-302.

23. Meyers RM, Valvassori G. Interpretation of anatomic variations of computed tomography scans of the sinuses: a surgeon's perspective. Laryngoscope. 1998; 108 (3): 422-425.

24. Stammberger H, Wolf G. Headaches and sinus disease: the endoscopic approach. Ann Otol Rhinol Laryngol Suppl. 1988; 134: 3-23.

25. Bolger WE, Butzin CA, Parsons DS. Paranasal sinus bony anatomic variations and mucosal abnormalities: CT analysis for endoscopic sinus surgery. Laryngoscope. 1991; 101 (1 Pt 1): $56-64$.

26. Cukurova I, Yaz A, Gumussoy M, Yigitbasi OG, Karaman Y. A patient presenting with concha bullosa in another concha bullosa: a case report. J Med Case Rep. 2012; 6: 87.

27. Stallman JS, Lobo JN, Som PM. The incidence of concha bullosa and its relationship to nasal septal deviation and paranasal sinus disease. AJNR Am J Neuroradiol. 2004; 25 (9): 1613-1618

28. Rahmati A, Ghafari R, AnjomShoa M. Normal variations of sphenoid sinus and the adjacent structures detected in cone beam computed tomography. J Dent (Shiraz). 2016; 17 (1): 32-37.

29. Güngör G, Okur N, Okur E. Uncinate process variations and their relationship with ostiomeatal complex: a pictorial essay of multidedector computed tomography (MDCT) findings. Pol J Radiol. 2016; 81: 173-180.

30. Tuli IP, Sengupta S, Munjal S, Kesari SP, Chakraborty S. Anatomical variations of uncinate process observed in chronic sinusitis. Indian J Otolaryngol Head Neck Surg. 2013; 65 (2): 157-161. 